\title{
Pneumococcal pneumonia prevalence among adults with severe acute respiratory illness in Thailand - comparison of Bayesian latent class modeling and conventional analysis
}

Ying Lu ${ }^{1 *}$ (D), Lawrence Joseph ${ }^{2}$, Patrick Bélisle ${ }^{3}$, Pongpun Sawatwong ${ }^{1}$, Anchalee Jatapai ${ }^{1,4}$, Toni Whistler ${ }^{1}$, Somsak Thamthitiwat ${ }^{1}$, Wantana Paveenkittiporn ${ }^{5}$, Supphacoke Khemla ${ }^{6}$, Chris A. Van Beneden? Henry C. Baggett ${ }^{8}$ and Christopher J. Gregory ${ }^{1,9}$

\begin{abstract}
Background: Determining the etiology of pneumonia is essential to guide public health interventions. Diagnostic test results, including from polymerase chain reaction (PCR) assays of upper respiratory tract specimens, have been used to estimate prevalence of pneumococcal pneumonia. However limitations in test sensitivity and specificity and the specimen types available make establishing a definitive diagnosis challenging. Prevalence estimates for pneumococcal pneumonia could be biased in the absence of a true gold standard reference test for detecting Streptococcus pneumoniae.

Methods: We conducted a case control study to identify etiologies of community acquired pneumonia (CAP) from April 2014 through August 2015 in Thailand. We estimated the prevalence of pneumococcal pneumonia among adults hospitalized for CAP using Bayesian latent class models (BLCMs) incorporating results of real-time polymerase chain reaction (qPCR) testing of upper respiratory tract specimens and a urine antigen test (UAT) from cases and controls. We compared the prevalence estimate to conventional analyses using only UAT as a reference test.

Results: The estimated prevalence of pneumococcal pneumonia was $8 \%$ (95\% Cl: 5-11\%) by conventional analyses. By BLCM, we estimated the prevalence to be $10 \%$ (95\% Crl: 7-16\%) using binary qPCR and UAT results, and $11 \%$ (95\% Crl: 7-17\%) using binary UAT results and qPCR cycle threshold (Ct) values.

Conclusions: BLCM suggests a $>25 \%$ higher prevalence of pneumococcal pneumonia than estimated by a conventional approach assuming UAT as a gold standard reference test. Higher quantities of pneumococcal DNA in the upper respiratory tract were associated with pneumococcal pneumonia in adults but the addition of a second specific pneumococcal test was required to accurately estimate disease status and prevalence. By incorporating the inherent uncertainty of diagnostic tests, BLCM can obtain more reliable estimates of disease status and improve understanding of underlying etiology.
\end{abstract}

Keywords: Pneumonia etiology, Pneumococcal pneumonia, Real-time polymerase chain reaction test, Urine antigen test, Pneumococcal density, Cycle threshold, Bayesian latent class

\footnotetext{
* Correspondence: ryluzhang@yahoo.com

${ }^{1}$ Division of Global Health Protection, Thailand Ministry of Public Health-US

Centers for Disease Control and Prevention Collaboration, Nonthaburi,

Thailand

Full list of author information is available at the end of the article
}

(c) The Author(s). 2019 Open Access This article is distributed under the terms of the Creative Commons Attribution 4.0 International License (http://creativecommons.org/licenses/by/4.0/), which permits unrestricted use, distribution, and

reproduction in any medium, provided you give appropriate credit to the original author(s) and the source, provide a link to the Creative Commons license, and indicate if changes were made. The Creative Commons Public Domain Dedication waiver (http://creativecommons.org/publicdomain/zero/1.0/) applies to the data made available in this article, unless otherwise stated. 


\section{Background}

An estimated 2.5 to 3 million pneumonia deaths occurred worldwide in 2010 [1]. Understanding the etiology of pneumonia is essential to guiding prevention strategies, improving clinical management, and minimizing the development of drug-resistance [2]. Pneumococcus is among the most important etiologies of hospitalized community-acquired pneumonia (CAP) among adults [3-6], but demonstrating Streptococcus pneumoniae as the etiology of pneumonia is challenging. In part this is because specimens from the site of infection (such as lung aspirates) are rarely collected nowadays for either clinical or research purposes. Pneumonia diagnosis currently tests largely on specimens not obtained directly from the lung (for example, blood, nasopharyngeal or oropharyngeal samples, urine, or induced sputum) which have imperfect sensitivity and specificity due to both the manner of collection and inherent to existing diagnostic tests such as culture or polymerase chain reaction (PCR) testing, despite advances in laboratory technology $[6,7]$.

Because it is rarely possible to definitively confirm pneumococcal pneumonia using existing diagnostic methods, this status is considered a "latent" variable. Latent class models (LCM) can link the latent variable with diagnostic tests results. This method has been used to estimate prevalence of pneumococcal pneumonia among adults with CAP in the United States (U.S.) and Kenya, incorporating results from multiple tests including PCR assays conducted on specimens from the upper respiratory tract or lung aspirate $[2,8]$. Cycle threshold $(\mathrm{Ct})$ values obtained from PCR are typically converted to a binary (positive or negative) result before further statistical analysis. However, when continuous or semi-quantitative test results such as $\mathrm{Ct}$ values are converted to a binary result, any potential association with the density of a detected pathogen is lost. The colonization density of $S$. pneumoniae has been shown in some but not all previous studies [9-13] to be associated with pneumococcal pneumonia.

During 2014 and 2015, the Thailand Ministry of Public Health (MOPH) and the U.S. Centers for Disease Control and Prevention (CDC) participated in a multicenter case-control study of the potential etiology of CAP among adults. In this study, real-time polymerase chain reaction (qPCR) testing for 16 viral and bacterial pathogens using the TaqMan array card (TAC) was performed on specimens collected from the upper respiratory tract. Similar to previous studies, $S$. pneumoniae was commonly detected but detection of $S$. pneumoniae from the upper respiratory tract can represent colonization in the absence of pneumococcal pneumonia, particularly among children [14-17].

We estimated the prevalence of pneumococcal pneumonia among adults aged 18 years and older who were hospitalized for CAP in Thailand during 2014 and 2015 using a Bayesian latent class model (BLCM) to incorporate $\mathrm{Ct}$ results of semi-quantitative pneumococcal qPCR testing of upper respiratory specimens and a second, more specific assay for $S$. pneumoniae, the urine antigen test (UAT) [18]. In addition, we compared the estimates from BLCM to those obtained by using qPCR results as a qualitative variable and to conventional analyses that considered UAT as a reference test for pneumococcal pneumonia. Secondary objectives included estimation of individual-level probability of pneumococcal pneumonia and evaluation of the test performance of UAT and qPCR of upper respiratory specimens to detect pneumococcal pneumonia.

\section{Methods}

\section{Study population and setting}

We identified eligible case-patients among persons $\geq 18$ years old hospitalized from April 2014 through August 2015 in one of four hospitals in Nakhon Phanom province in Northeast Thailand: Nakhon Phanom, Nakae, Srisongkharm, and Tautane. We defined CAP as illness meeting WHO's global influenza surveillance definition for severe acute respiratory illness (SARI): an acute respiratory infection and history of fever or measured fever of $\geq 38 \mathrm{C}^{\circ}$ and cough with onset within the last 7 days requiring hospitalization [19]. A potential case was excluded if he/she had been hospitalized for any cause in the previous 14 days or had an episode of pneumonia or been previously enrolled in the study within the past 30 days. We randomly selected controls from dental clinics in these hospitals to represent the population in the same residential areas as case-patients. Controls were frequency matched by age group (18-49, 50-64 and $\geq 65$ years old) and enrolled within 2 weeks of case enrollment in order to control for pathogen seasonality. A control was excluded if he/she had been hospitalized for any cause in the previous 14 days, had an episode of pneumonia or were previously enrolled in the study within the past 30 days, or had any current symptom of acute respiratory infection. To maximize case enrollment while maintaining the ability to detect a statistical difference among cases and controls for viral and bacterial etiologies given the limited number of TAC cards available, we selected a 5:2 case to control target ratio.

\section{Specimen and data collection}

Nasopharyngeal (NP) and oropharyngeal (OP) swabs and a urine specimen were collected from all enrolled cases and controls. A standard case report form was completed to capture demographic characteristics, medical history, clinical signs and symptoms, date of illness onset, hospital course and outcome including receipt of antibiotics prior to specimen collection. NP/OP swabs 
were collected into $3 \mathrm{ml}$ of universal transport medium (UTM) at the study sites, aliquoted, frozen and transported to the central laboratory where they were stored at $-70^{\circ} \mathrm{C}$. Total nucleic acid (TNA) extraction from 200 ul of NP/OP swabs UTM was performed using the NucliSens easyMAG system (BioMerieux, Marcy l'Etoile, France), with a final elution volume of 100 ul. The $S$. pneumoniae primers and probes on the TAC were against the autolysin $(L y t A)$ gene from previously published assays [20]. Briefly, $46 \mu \mathrm{l}$ specimen TNA was used in the AgPath-ID One-Step RT-PCR kit, (Applied Biosystems, CA, USA). A no-template control and a positive control consisting of combined RNA transcripts, generated as previously described [21], were included on each TAC along with up to six patient specimens. Reaction mixtures were loaded into the individual portals, and the card was centrifuged twice at $335 \times \mathrm{g}$ for $1 \mathrm{~min}$ and sealed to close the reaction wells. All TACs were run on an Applied Biosystems ViiA7 real-time PCR instrument (Life Technologies) using the following cycling conditions: $45^{\circ} \mathrm{C}$ for $10 \mathrm{~min}, 94^{\circ} \mathrm{C}$ for $10 \mathrm{~min}, 45$ cycles of $94{ }^{\circ} \mathrm{C}$ for $30 \mathrm{~s}$, and $60^{\circ} \mathrm{C}$ for $60 \mathrm{~s}$. The Ct value represents the number of amplification cycles needed before the pathogen-specific nucleic acid was detected. Therefore, higher $\mathrm{Ct}$ values indicate lower amounts of the target organism DNA in the extracted sample.

Urine was tested in the hospital laboratory by the Binax NOW ${ }^{\circ}$ S. pneumoniae Antigen Card immunochromatographic test. Positive and negative controls provided by the manufacturer were run daily. Published estimates of the sensitivity and specificity of this assay from a meta-analysis on UAT performance are 0.75 (95\% CI: 0.71-0.79) and 0.95 (95\% CI: 0.92-0.98) [22].

\section{Statistical analysis}

To estimate the prevalence of pneumococcal pneumonia in cases, both conventional analyses with UAT as the reference test and BLCMs without referencing a gold standard test were conducted.

\section{Conventional analyses}

For the primary conventional analysis, UAT was assumed to have $100 \%$ sensitivity and specificity; patients with positive UAT results were considered true pneumococcal pneumonia cases and those with negative UAT results were considered non-pneumococcal pneumonia cases. Prevalence was estimated using the proportion of UAT positive (a binomial proportion), and 95\% confidence interval $(\mathrm{CI})$ was calculated based on standard error of the binomial proportion. The mean $\mathrm{Ct}$ value among pneumococcal pneumonia cases was calculated as the mean of $\mathrm{Ct}$ values among UAT-positive patients with a positive $\mathrm{qPCR}$ result. The mean $\mathrm{Ct}$ value in non-pneumococcal pneumonia was calculated similarly among UAT-negative participants. We also conducted an adjusted conventional analysis to obtain a prevalence estimate adjusted by the reported sensitivity of UAT from previous studies [22]. The prevalence estimate from the primary conventional analysis model was divided by $75 \%$. These analyses were done in RStudio (Version 1.0.136 -@ 2009-2016 RStudio, Inc.)

\section{Bayesian latent class modelling (BLCM)}

BLCM models were run using either binary or continuous qPCR results; both models include UAT results. We used the model by Joseph et al. [23] to analyze qPCR results as a binary variable using the Bayes Diagnostic Tests program available from http://www.medicine.mcgill.ca/epidemiology/Joseph/software/Diagnostic-Testing. html. A model based on Weichenthal et al. [24] was created for utilizing qPCR Ct values. Parameters included in the models and methods for the selection of prior distributions of those parameters and sensitivity analyses are described in the Additional file 1: Appendix. We ran 20,000 iterations of the Gibbs sampler algorithm after burn-in of 5000 iterations. All model runs were checked for convergence using the convergence diagnostics provided by the WinBUGS v. 1.4.3 (Imperial College and MRC, UK, see the WinBUGS code we used, provided in the Appendix), via examination of the history plots of all parameters.

\section{Results}

From April 2014 to August 2015, 5171 hospitalized patients were screened for CAP; among 462 eligible patients, 357 (77.3\%) were enrolled. During the same period, 255 healthy individuals were screened as potential controls; 238 were eligible and 217 (91.2\%) enrolled. Among case-patients, $45 \%$ (161/357) were male with a mean age of 62 years (standard deviation SD: 18 years). Controls were 37\% (80/ 217) male with a mean age of 58 years (SD: 16 years).

Among 357 case-patients, 32\% (114/357) had a NP/ OP specimen positive for $S$. pneumoniae by qPCR compared to 26\% (57/217) of controls. Of 353 case-patients also tested by UAT, $8 \%(27 / 353)$ were positive compared to $0.5 \%(1 / 217)$ of controls. While no controls were positive by both assays, $7.4 \%(26 / 353)$ of case-patients were UAT and qPCR positive (Table 1). In qPCR-positive samples, the mean Ct value among case-patients was lower (29.8 [SD: 4.9]) than that among controls (33.0 [SD: 4.8]); however, the distribution of case and control Ct values overlapped (Fig. 1). UAT-positive case-patients had a mean Ct value of 25.6 (SD: 3.3) compared to 31 (SD: 4.7) in UAT negative case-patients. Seventy-five percent $(266 / 357)$ of case-patients received antibiotics prior to NP/OP swab; 35\% (93/266) of these case-patients were qPCR positive, with a mean $\mathrm{Ct}$ value of 29.9 (SD: 4.7). Among 91 case-patients who did not 
Table 1 S. pneumoniae detection by real-time polymerase chain reaction (qPCR) on nasopharyngeal and oropharyngeal specimens and urine antigen test (UAT) among case-patients with communityacquired pneumonia in Nakhon Phanom, Thailand, April 2014 August 2015

\begin{tabular}{lllll}
\hline & & UAT & & Total \\
\cline { 3 - 4 } & & Positive & Negative & \\
\hline qPCR & Positive & 26 & 85 & $111(31 \%)$ \\
& Negative & 1 & 241 & 242 \\
Total & & $27(8 \%)$ & 326 & $353^{*}$ \\
\hline
\end{tabular}

*Among 357 cases, four cases did not have UAT test done

receive antibiotics, $23 \%(21 / 91)$ were GPCR positive with a mean Ct value of 28.9 (SD: 5.7).

\section{Conventional analysis}

In conventional analysis, prevalence of pneumococcal pneumonia among cases was $8 \%$ (95\% CI: 5-11\%). The positive predictive value (PPV) of UAT was $100 \%$. The prevalence was $10 \%$ (95\% CI: 7-15\%) with the adjusted analysis, which considered UAT to be $75 \%$ sensitive (Table 2).

\section{BLCM with qualitative $\mathrm{qPCR}$ results}

The estimated pneumococcal pneumonia prevalence among case-patients was $10 \%$ (95\% CrI: 7-16\%) with a PPV for pneumococcal pneumonia given a positive qPCR of $31 \%$ (95\% CrI: 20-44\%). (Table 2). The specificity of UAT was estimated as $99.5 \%$ (95\% CrI: 98.599.9\%) and the sensitivity as $72 \%$ (95\% CrI: $53-87 \%$ ). The specificity of qPCR was estimated as 75\% (71-79\%) and the sensitivity as 96\% (95\% CrI: $83-100 \%)$. The PPV for pneumococcal pneumonia given a positive UAT was 94\% (95\% CrI: 83-99\%).

\section{BLCM with continuous $\mathrm{qPCR}$ results}

The prevalence of pneumococcal pneumonia in case-patients was estimated at 11\% (95\% CrI: 7-17\%). Estimates of the sensitivity, specificity and PPV of UAT using continuous qPCR Ct results were similar to those generated from the model using qualitative qPCR results (Table 2).

Based on estimates of individual probability among qPCR-positive cases, the probability for a case-patient having pneumococcal pneumonia ranged from 0 (95\% CrI 0-0\%) with a $\mathrm{Ct}$ value of 42 and a negative UAT result to $100 \%$ (95\% CrI 99-100\%) with a Ct value of 20.9 and a positive UAT result (Fig. 2). The one case-patient who was qPCR negative and UAT positive had a probability of pneumococcal pneumonia of $49 \%$ with a wide range of uncertainty (95\% CrI: 3-92\%).

\section{Sensitivity analysis}

After widening the possible ranges of prior values for parameters derived from controls while keeping the same mean values, the prevalence of pneumococcal pneumonia was estimated as $11 \%$ (95\% CrI: $7-17 \%)$ in the model using binary qPCR results and 11\% (95\% CrI: 7-16\%) for the model using continuous $\mathrm{Ct}$ values.

\section{Discussion}

In this analysis, we estimated the prevalence of pneumococcal pneumonia among adults hospitalized with CAP in Thailand and compared the estimates between conventional analyses and BLCMs. Incorporating $\mathrm{qPCR} C \mathrm{Ct}$

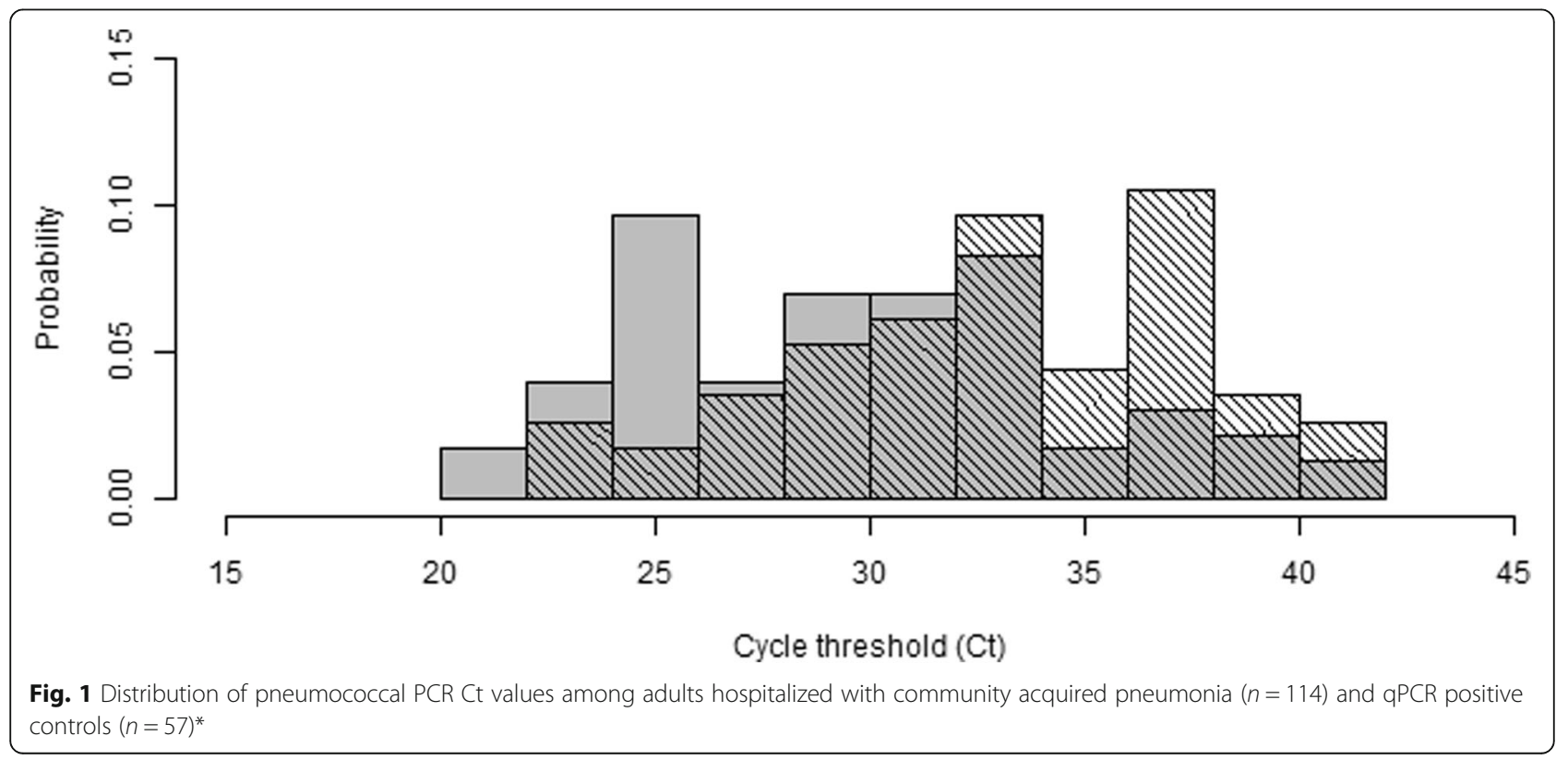


Table 2 Prevalence of pneumococcal pneumonia (PPN) in case-patients and performance of real-time polymerase chain reaction (qPCR) and urine antigen test (UAT): conventional analysis with UAT as a gold standard vs. Bayesian latent class models (BLCM) $(n=353)$

\begin{tabular}{|c|c|c|c|c|}
\hline & \multicolumn{2}{|l|}{$\begin{array}{l}\text { Conventional analyses } \\
\text { Mean }(95 \% \mathrm{Cl})\end{array}$} & \multicolumn{2}{|l|}{$\begin{array}{l}\text { BLCM } \\
\text { Median (95\% Crl) }\end{array}$} \\
\hline & Primary & Adjusted & Binary qPCR model & Continuous qPCR model \\
\hline & $\begin{array}{l}27 \text { UAT positives considered } \\
\text { true positives }\end{array}$ & $\begin{array}{l}\text { UAT sensitivity is } 75 \% \\
\text { and specificity is } 100 \%\end{array}$ & $\mathrm{qPCR}$ and UAT & $\mathrm{qPCR}$ and UAT \\
\hline Prevalence & $8 \%(5-11 \%)$ & $10 \%(7-15 \%)$ & $10 \%(7-16 \%)$ & $11 \%(7-17 \%)$ \\
\hline Sensitivity of qPCR & $96 \%(81-100 \%)$ & & $96 \%(83-100 \%)$ & \\
\hline Specificity of qPCR & $74 \%(69-79 \%)$ & & $75 \%(71-79 \%)$ & \\
\hline PPV of $\mathrm{qPCR}$ & $23 \%(16-32 \%)$ & & $31 \%(20-44 \%)$ & \\
\hline Mean qPCR Ct among PPN & $25.6(24.2-26.9)$ & & & $25.7(24.6-27)$ \\
\hline Mean qPCR among Non-PPN & $31(30-32)$ & & & $32.4(31.4-33.4)$ \\
\hline Sensitivity of UAT & $100 \%$ (assumed) & 75\% (published) & $72 \%(53-87 \%)$ & $67 \%(51-83 \%)$ \\
\hline Specificity of UAT & 100\% (assumed) & $100 \%$ (assumed) & $99.5 \%(98.5-99.9 \%)$ & $99.5 \%(98.6-99.9 \%)$ \\
\hline PPV of UAT & $100 \%$ & $100 \%$ & $94 \%$ (83-99\%) & $95 \%(83-99 \%)$ \\
\hline Probability of PPV of UAT $<90 \%$ & 0 & 0 & $18 \%$ & $17 \%$ \\
\hline
\end{tabular}

values for pneumococcal DNA in NP/OP specimens allowed the probability of pneumococcal pneumonia in individual patients to also be estimated. Our study demonstrated the impact of integrating results from multiple assays and accounting for imperfect test performance on the estimated probability and prevalence of pneumococcal pneumonia.

The prevalence estimated from BLCM was $>25 \%$ higher than the prevalence estimated from the primary conventional analysis ( $10 \%$ or $11 \%$ vs. $8 \%)$. Recent investigations of pneumococcal pneumonia have attempted to take imperfect test performance into account during analysis, due to increased evidence of the imperfectness of the usual reference tests such as UAT and blood culture [25]. The adjusted conventional analysis, although simply based on a fixed value of sensitivity and specificity of UAT, here provided a similar prevalence estimate and uncertainty intervals as the results from BLCM. However although not the case here, conventional analyses may provide potentially misleading estimates of disease prevalence since they neither take into account the uncertainty about these fixed values nor easily incorporate results from multiple imperfect tests. In BLCM, estimates of test performance gathered from the literature as well as test results from controls can be incorporated and quantified as prior distributions. Therefore, the prevalence estimate from BLCM is more robust and utilizes the comprehensive information provided by the case control study design. Compared to prevalence estimates based on conventional analyses using UAT in Thailand (3-4\%) [18, 26], our estimates using BLCM or conventional analyses indicate a higher prevalence of pneumococcus in adults in Thailand than previously documented.

Using qPCR Ct values allowed estimation of the probability of pneumococcal pneumonia for each individual case-patient (Fig. 2). In contrast the use of qualitative qPCR values estimated the PPV for pneumococcal pneumonia for all qPCR positive case-patients to be $31 \%$ (95\% CrI: 20-44\%) (Table 2). As has been seen in previous studies, higher density of pneumococcus in the upper airway, as indicated by a lower $\mathrm{Ct}$ value, was associated with a higher likelihood of pneumococcal pneumonia [9-13].

The prevalence point estimate and 95\% CrIs were similar for the BLCM using binary qPCR and continuous qPCR results when UAT results were included. This finding suggests, at a population level, a much greater predictive value of incorporating UAT testing for pneumococcal pneumonia than the added value of utilizing qPCR Ct values rather than binary qPCR results. For individual case-patients with lower qPCR Ct values and a positive UAT or higher $\mathrm{Ct}$ values and negative UAT results, 95\% CrIs for the probability of pneumococcal pneumonia were narrow, indicating high certainty in the point estimates. In our study, the addition of the UAT result was necessary to determine pneumococcal pneumonia with a reasonable degree of certainty.

However, the potential for misclassification of disease status by UAT remained as demonstrated by the estimated $~ 17 \%$ likelihood that the PPV of UAT is $<90 \%$ in BLCM. The specificity of UAT by BLCM was estimated as close to the prior estimate of $100 \%$ (99.5\%; 98.699.9\%) but there remained substantial uncertainty around the sensitivity estimates, which ranged from 51 


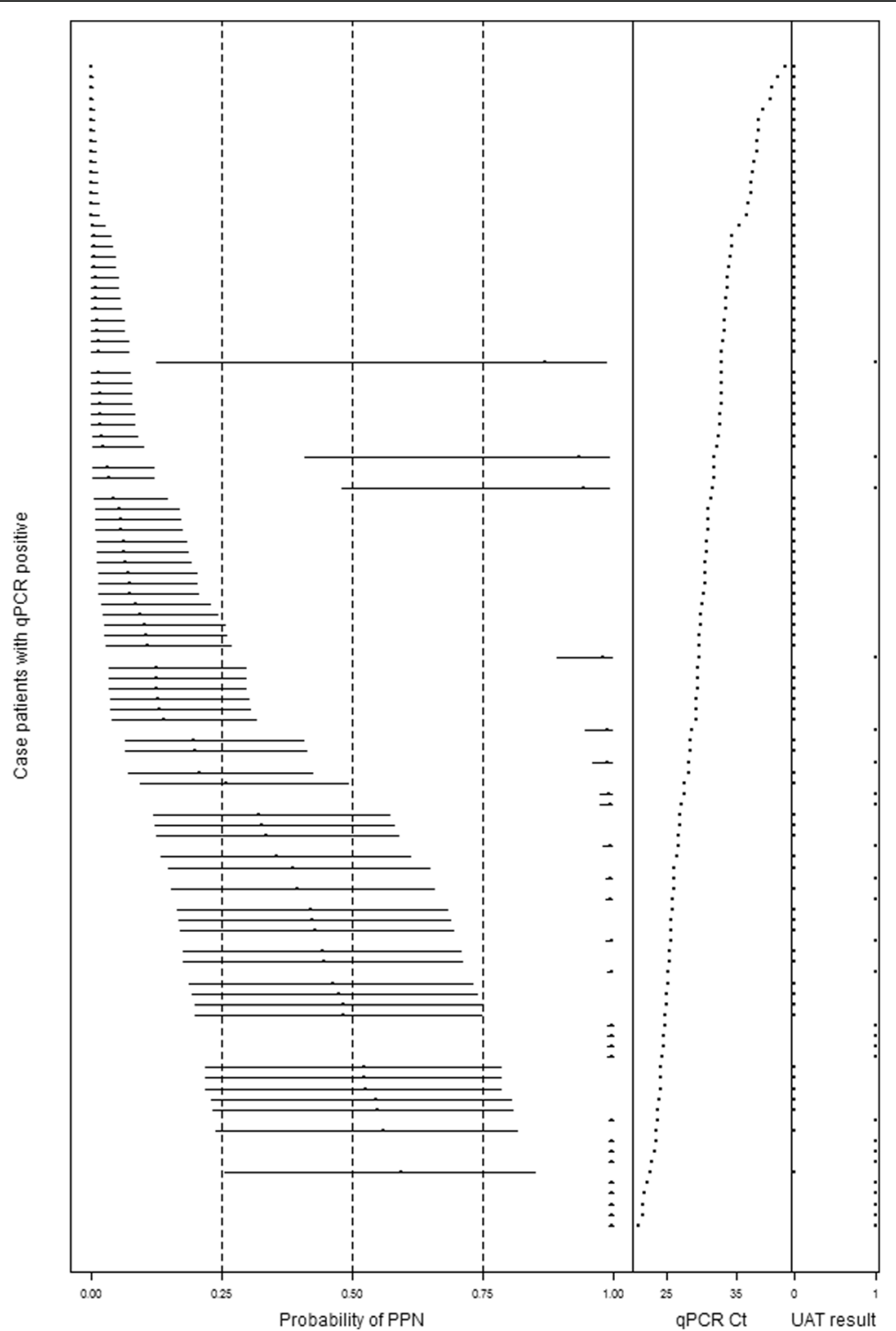

Fig. 2 Individual probability of pneumococcal pneumonia (PPN) given pneumococcal Ct value from real-time polymerase chain reaction (qPCR) and urine antigen test (UAT) results in qPCR positive case-patients $(n=111)$

to $83 \%$. These estimates were not updated much compared to the range (50-90\%) selected for prior sensitivity of UAT [22] [2, 27-31]. This may indicate that our study data did not add significant new information regarding the limited known sensitivity of UAT. This wide uncertainty range for the sensitivity of UAT supports not utilizing a fixed value for UAT sensitivity to estimate disease prevalence, as would be the case when simply adjusting results of conventional analysis by a set value to account for imperfect sensitivity.

BLCM can be useful to obtain the best possible estimates [23] despite having multiple unknown parameters; however, the posterior estimates from these models depend heavily on the prior distributions of these parameters used. Our carefully designed case-control study provided an important source of information for some prior distributions. To ascertain the robustness of the estimates to this assumption, we performed a sensitivity analysis to account for the possibility that qPCR or UAT results differed between controls and non-pneumococcal pneumonia case-patients; however, the prevalence estimates and all other parameters were almost identical to the main analyses (data not shown). Our estimates of prevalence from BLCM could also be further improved with better knowledge of the sensitivity of urinary antigen assay for disease detection. Recently, the use of a serotype-specific urinary antigen detection assays was demonstrated to substantially increase the detection of pneumococcal pneumonia 
among adult patients with CAP in the U.S. [25], suggesting the sensitivity of the conventional UAT might be more limited than previously appreciated. Further efforts to more precisely define the true sensitivity of the UAT would be beneficial to provide better prior distributions to be used in future Bayesian analyses. Another limitation was the assumption that the results from qPCR and UAT were conditionally independent of the true pneumococcal pneumonia status. If the test results were conditionally dependent, additional parameters such as correlation coefficients between the sensitivities and specificities of the tests would need to be incorporated into the model to generate reasonable estimates. An inherent limitation in qPCR testing of upper respiratory specimens is the inability to control for the quantity of specimen on each swab. The qPCR-positive swabs with more voluminous specimen could have lower $\mathrm{Ct}$ values even if the pathogen density was not actually higher than in a patient whose swab had lower volume. The density of host DNA offers some indication of specimen volume, and in our data, the host DNA Ct values varied little: the mean $\mathrm{Ct}$ value among case-patients was 24 (SD: 1.7) and among controls was 24 (SD: 1.4). Therefore, we did not attempt to control for specimen volume with the human DNA Ct. The potential influence of prior antibiotic use on pneumococcal pneumonia estimates is of interest but was not able to be examined in the BLCM models due to the relatively small number of case-patients who had not previously received antibiotics in our study population.

\section{Conclusions}

Using BLCM to integrate results from two independent assays and accounting for imperfect test performance, we found that $11 \%$ (95\% CrI: $7-17 \%$ ) of CAP among adults in Thailand was associated with pneumococcus, a higher prevalence than previously estimated from a similar population. Our findings support the hypothesis that upper airway pneumococcal DNA density as based on qPCR Ct value is associated with pneumococcal pneumonia in adults but requires the inclusion of an additional specific pneumococcal test to accurately estimate disease status and prevalence. BLCM can help obtain superior estimates of population disease status when multiple independent test results exist in the absence of a true gold standard reference test in order to inform vaccine cost effectiveness analyses and other disease prevention strategies.

\section{Additional file}

Additional file 1: Appendix. Bayesian latent class modeling (BLCM).

The Bayesian latent class model, prior selection and sensitivity analyses used in this manuscript. (DOCX $34 \mathrm{~kb}$ )

\section{Abbreviations}

BLCM: Bayesian latent class model; CAP: community acquired pneumonia; CDC: Centers for Disease Control and Prevention; Cl: confidence interval; Crl: credible interval; Ct: cycle threshold; NP: nasopharyngeal;

OP: oropharyngeal; PCR: polymerase chain reaction; PPN: pneumococcal pneumonia; PPV: positive predicative value; QPCR: real time polymerase chain reaction; SARI: severe acute respiratory illness; SD: standard deviation;

TNA: Total nucleic acid; UTM: universal transport medium

\section{Acknowledgements}

We thank Ornuma Sangwichian, Apiwat Lapamnouysup, Prasong Srisaengchai and Pornpak Khunatorn from the Division of Global Health Protection, Thailand Ministry of Public Health-US Centers for Disease Control and Prevention Collaboration, Nonthaburi, Thailand and the provincial health officials from Nakhon Phanom province.

\section{Funding}

The funding was provided by the U.S. Centers for Disease Control and Prevention. The findings and conclusions in this report are those of the authors and do not necessarily represent the official position of the CDC. All authors report no conflicts of interest relevant to this article.

\section{Availability of data and materials}

The data that support the findings of this study are available from Thailand Ministry of Public Health but restrictions apply to the availability of these data, which were used by permission for the current study, and so are not publicly available. Data are however available from the authors upon reasonable request and with permission of Thailand Ministry of Public Health.

Authors' contributions

$Y L, L, H C B$ and $C J G$ analyzed and interpreted the patient data and were major contributors in writing the manuscript. CAVB, HCB and CJG designed this study and were major reviewers in draft manuscript development. AJ collected data and PS performed qPCR test. $\amalg$ and PB created Bayesian latent class models. TW, WP, ST and SK advised on draft manuscript reviews. All authors read and approved the final manuscript.

\section{Ethics approval and consent to participate}

The Thailand MOPH ethical review committee (ERC) reviewed and approved the study protocol. The U.S. Centers for Disease Control and Prevention (CDC)'s Human Research Protection Office approved the study based on reliance on Thailand ERC's approval. The consent obtained from study participants was written.

Consent for publication

Not applicable.

\section{Competing interests}

The authors declare that they have no competing interests.

\section{Publisher's Note}

Springer Nature remains neutral with regard to jurisdictional claims in published maps and institutional affiliations.

\footnotetext{
Author details

'Division of Global Health Protection, Thailand Ministry of Public Health-US Centers for Disease Control and Prevention Collaboration, Nonthaburi, Thailand. ${ }^{2}$ Department of Epidemiology and Biostatistics, McGill University, Montreal, Canada. ${ }^{3}$ Centre Hospitalier de I'Universite de Montreal,Montreal, Montreal, Canada. ${ }^{4}$ Office of Public Health, Regional Development Mission for Asia, US Agency for International Development, Bangkok, Thailand. ${ }^{5}$ Department of Medical Sciences, Ministry of Public Health, National Institute of Health, Nonthaburi, Thailand. ${ }^{6}$ Nakhon Phanom Provincial Hospital, Ministry of Public Health, Nakhon Phanom, Thailand. ${ }^{7}$ Division of Bacterial Diseases, National Center for Immunization and Respiratory Disease, US Centers for Disease Control and Prevention, Atlanta, GA, USA. ${ }^{8}$ Division of Global Health Protection, US Centers for Disease Control and Prevention, Atlanta, GA, USA. ${ }^{9}$ Present affiliation: Division of Vector-Borne Diseases, US Centers for Disease Control and Prevention, Fort Collins, CO, USA.
} 
Received: 22 August 2018 Accepted: 7 May 2019

Published online: 15 May 2019

\section{References}

1. Lozano R, Naghavi M, Foreman K, Lim S, Shibuya K, Aboyans V, et al. Global and regional mortality from 235 causes of death for 20 age groups in 1990 and 2010: a systematic analysis for the global burden of disease study 2010 Lancet. 2012;380(9859):2095-128.

2. Butler JC, Bosshardt SC, Phelan M, Moroney SM, Tondella ML, Farley MM, et al. Classical and latent class analysis evaluation of sputum polymerase chain reaction and urine antigen testing for diagnosis of pneumococcal pneumonia in adults. J Infect Dis. 2003;187(9):1416-23.

3. Song JY, Eun BW, Nahm MH. Diagnosis of pneumococcal pneumonia: current pitfalls and the way forward. Infect Chemother. 2013;45(4):351-66.

4. Holter JC, Muller F, Bjorang O, Samdal HH, Marthinsen JB, Jenum PA, et al. Etiology of community-acquired pneumonia and diagnostic yields of microbiological methods: a 3-year prospective study in Norway. BMC Infect Dis. 2015;15:64.

5. LeBlanc JJ, EISherif M, Ye L, MacKinnon-Cameron D, Li L, Ambrose A, et al. Burden of vaccine-preventable pneumococcal disease in hospitalized adults: a Canadian immunization research network (CIRN) serious outcomes surveillance (SOS) network study. Vaccine. 2017:35(29):3647-54.

6. Feikin DR, Hammitt LL, Murdoch DR, O'Brien KL, Scott JAG. The Enduring Challenge of Determining Pneumonia Etiology in Children: Considerations for Future Research Priorities. Clin Infect Dis. 2017;64(suppl_3):S188-S96.

7. Hammitt LL, Feikin DR, Scott JAG, Zeger SL, Murdoch DR, O'Brien KL, et al. Addressing the Analytic Challenges of Cross-Sectional Pediatric Pneumonia Etiology Data. Clin Infect Dis. 2017;64(suppl_3):S197-204.

8. Jokinen J, Scott JA. Estimating the proportion of pneumonia attributable to pneumococcus in Kenyan adults: latent class analysis. Epidemiology. 2010; 21(5):719-25.

9. Alpkvist H, Athlin S, Naucler P, Herrmann B, Abdeldaim G, Slotved HC, et al. Clinical and microbiological factors associated with high nasopharyngeal pneumococcal density in patients with pneumococcal pneumonia. PLoS One. 2015:10(10):e0140112.

10. Wolter N, Tempia S, Cohen C, Madhi SA, Venter M, Moyes J, et al. High nasopharyngeal pneumococcal density, increased by viral coinfection, is associated with invasive pneumococcal pneumonia. J Infect Dis. 2014; 210(10):1649-57.

11. Albrich WC, Madhi SA, Adrian PV, van Niekerk N, Mareletsi T, Cutland C, et al. Use of a rapid test of pneumococcal colonization density to diagnose pneumococcal pneumonia. Clin Infect Dis. 2012;54(5):601-9.

12. Baggett HC, Watson NL, Deloria Knoll M, Brooks WA, Feikin DR, Hammitt LL, et al. Density of Upper Respiratory Colonization With Streptococcus pneumoniae and Its Role in the Diagnosis of Pneumococcal Pneumonia Among Children Aged $<5$ Years in the PERCH Study. Clin Infect Dis. 2017; 64(suppl_3):S317-S27.

13. Deloria Knoll M, Morpeth SC, Scott JAG, Watson NL, Park DE, Baggett HC, et al. Evaluation of Pneumococcal Load in Blood by Polymerase Chain Reaction for the Diagnosis of Pneumococcal Pneumonia in Young Children in the PERCH Study. Clin Infect Dis. 2017;64(suppl_3):S357-S67.

14. Hill PC, Townend J, Antonio M, Akisanya B, Ebruke C, Lahai G, et al. Transmission of Streptococcus pneumoniae in rural Gambian villages: a longitudinal study, Clin Infect Dis. 2010:50(11):1468-76.

15. Tenenbaum T, Franz A, Neuhausen N, Willems R, Brade J, Schweitzer-Krantz $\mathrm{S}$, et al. Clinical characteristics of children with lower respiratory tract infections are dependent on the carriage of specific pathogens in the nasopharynx. Eur J Clin Microbiol Infect Dis. 2012;31(11):3173-82.

16. Abdullahi O, Nyiro J, Lewa P, Slack M, Scott JA. The descriptive epidemiology of Streptococcus pneumoniae and Haemophilus influenzae nasopharyngeal carriage in children and adults in Kilifi district, Kenya. Pediatr Infect Dis J. 2008;27(1):59-64.

17. Simell B, Auranen K, Kayhty H, Goldblatt D, Dagan R, O'Brien KL, et al. The fundamental link between pneumococcal carriage and disease. Expert Rev Vaccines. 2012;11(7):841-55.

18. Piralam B, Tomczyk SM, Rhodes JC, Thamthitiwat S, Gregory CJ, Olsen SJ, et al. Incidence of pneumococcal pneumonia among adults in rural Thailand, 2006-2011: implications for pneumococcal vaccine considerations. Am J Trop Med Hyg. 2015;93(6):1140-7.

19. WHO Global Surveillance Manual on Influenza. 2011:6.
20. Kodani M, Yang G, Conklin LM, Travis TC, Whitney CG, Anderson LJ, et al. Application of TagMan low-density arrays for simultaneous detection of multiple respiratory pathogens. J Clin Microbiol. 2011;49(6):2175-82.

21. Kodani M, Winchell JM. Engineered combined-positive-control template for real-time reverse transcription-PCR in multiple-pathogen-detection assays. J Clin Microbiol. 2012;50(3):1057-60.

22. Horita N, Miyazawa N, Kojima R, Kimura N, Inoue M, Ishigatsubo Y, et al. Sensitivity and specificity of the Streptococcus pneumoniae urinary antigen test for unconcentrated urine from adult patients with pneumonia: a metaanalysis. Respirology (Carlton, Vic). 2013;18(8):1177-83.

23. Joseph L, Gyorkos TW, Coupal L. Bayesian estimation of disease prevalence and the parameters of diagnostic tests in the absence of a gold standard. Am J Epidemiol. 1995;141(3):263-72.

24. Weichenthal S, Joseph L, Belisle P, Dufresne A. Bayesian estimation of the probability of asbestos exposure from lung fiber counts. Biometrics. 2010; 66(2):603-12.

25. Wunderink RG, Self WH, Anderson EJ, Balk R, Fakhran S, Courtney DM, et al. Pneumococcal community-acquired pneumonia detected by serotype-specific urinary antigen detection assays. Clin Infect Dis. 2018;66(10):1504-10.

26. Olsen SJ, Thamthitiwat S, Chantra S, Chittaganpitch M, Fry AM, Simmerman $J M$, et al. Incidence of respiratory pathogens in persons hospitalized with pneumonia in two provinces in Thailand. Epidemiol Infect. 2010;138(12): $1811-22$.

27. Marcos MA, Jimenez de Anta MT, de la Bellacasa JP, Gonzalez J, Martinez E, Garcia E, et al. Rapid urinary antigen test for diagnosis of pneumococcal community-acquired pneumonia in adults. Eur Respir J. 2003:21(2):209-14

28. Murdoch DR, Laing RT, Cook JM. The NOW S. Pneumoniae urinary antigen test positivity rate 6 weeks after pneumonia onset and among patients with COPD. Clin Infect Dis 2003;37(1):153-154.

29. Klugman KP, Madhi SA, Albrich WC. Novel approaches to the identification of Streptococcus pneumoniae as the cause of community-acquired pneumonia. Clin Infect Dis. 2008;47(Suppl 3):S202-6.

30. Smith MD, Derrington P, Evans R, Creek M, Morris R, Dance DA, et al. Rapid diagnosis of bacteremic pneumococcal infections in adults by using the Binax NOW Streptococcus pneumoniae urinary antigen test: a prospective, controlled clinical evaluation. J Clin Microbiol. 2003;41(7):2810-3.

31. Werno AM, Murdoch DR. Medical microbiology: laboratory diagnosis of invasive pneumococcal disease. Clin Infect Dis. 2008;46(6):926-32.
Ready to submit your research? Choose BMC and benefit from:
- fast, convenient online submission
- thorough peer review by experienced researchers in your field
- rapid publication on acceptance
- support for research data, including large and complex data types
- gold Open Access which fosters wider collaboration and increased citations
- maximum visibility for your research: over $100 \mathrm{M}$ website views per year
At $\mathrm{BMC}$, research is always in progress.
Learn more biomedcentral.com/submissions 